\title{
Correction to: Shared Images and Camera Fingerprinting May Lead to Privacy Issues
}

Rahimeh Rouhi, Flavio Bertini, and Danilo Montesi

\section{Correction to:}

Chapter 1 in: T. Bourlai et al. (eds.), Securing Social Identity in Mobile Platforms, Advanced Sciences and Technologies for Security Applications, https://doi.org/10.1007/978-3-03039489-9_1

The original version of the book was inadvertently published with an error in the name of chapter author (Flavio Bertini) in the chapter opening page of the first chapter in Part I. 